\title{
The Arens Algebras of Vector-Valued Functions
}

\author{
I. G. Ganiev ${ }^{1,2}$ and O. I. Egamberdiev ${ }^{1,2}$ \\ ${ }^{1}$ Department of Science in Engineering, Faculty of Engineering, International Islamic University Malaysia, P.O. Box 10, \\ 50728 Kuala Lumpur, Malaysia \\ ${ }^{2}$ Department of Algebra and Functional Analysis, National University of Uzbekistan, Vuzgorodok, 100174 Tashkent, Uzbekistan \\ Correspondence should be addressed to I. G. Ganiev; inam@iium.edu.my
}

Received 4 September 2013; Accepted 5 December 2013; Published 23 February 2014

Academic Editor: Dragan Djordjevic

Copyright (C) 2014 I. G. Ganiev and O. I. Egamberdiev. This is an open access article distributed under the Creative Commons Attribution License, which permits unrestricted use, distribution, and reproduction in any medium, provided the original work is properly cited.

A class of Arens algebras of vector Banach algebra valued functions is considered. It is shown that Arens algebra of vector-valued functions is complete locally convex metrizable algebra.

\section{Introduction}

Let $(\Omega, \Sigma, \mu)$ be a measure space with finite measure $\mu$ and let $L^{p}=L^{p}(\Omega, \Sigma, \mu)$ be the Banach space of all measurable functions $f$ on $(\Omega, \Sigma, \mu)$ such that $|f|_{p}=\left(\int_{\Omega}|f|^{p} d \mu\right)^{1 / p}<\infty$, $(1 \leq p<\infty)$. In [1] the set

$$
L^{\omega}=\bigcap_{p \geq 1} L^{p}
$$

is introduced and it is shown that $L^{\omega}$ is locally convex algebra in topology $\tau$ generated by the norms $\|\cdot\|_{p}$. Such algebras are called Arens algebras. In [2] it is shown that $\left(L^{\omega}, \tau\right)$ is $G B^{*}$ algebra. Properties of algebra $L^{\omega}$ are considered in $[3,4]$. Moreover, it is shown that dual of $L^{\omega}$ is $\bigcup_{1<q \leq \infty} L^{q}$ and $L^{\omega}$ is reflexive. In [5] a class of noncommutative analogue of Arens algebra $L^{\omega}(M, \varphi)$ associated with semifinite von Neumann algebra and semifinite trace $\varphi$ is introduced and in [6] it is shown that $L^{\omega}(M, \varphi)$ is $E W^{*}$ algebra. In [6-8], properties of noncommutative Arens algebras are considered. Noncommutative Arens algebras $L^{\omega}(M, \Phi)$ associated with semifinite von Neumann algebra and center-valued trace $\Phi$ are introduced in [9] and in [10] they are generalized for the case when $\Phi$ is the Maharam trace.
Let $L^{p}(\Omega, X)$ be of Bochner maps from $\Omega$ into a unital Banach algebra $\left(X,\|\cdot\|_{X}\right)$, integrable with degree $p, 1 \leq p<$ $\infty$, that is, $L^{p}$ space of vector-valued functions, and

$$
L^{\omega}(\Omega, X)=\bigcap_{p \geq 1} L^{p}(\Omega, X) .
$$

In the present paper we prove that $L^{\omega}(\Omega, X)$ is complete locally convex metrizable algebra, which we call Arens algebra of vector-valued functions. It is shown that if dual of $X$ has Radon-Nikodym property, then dual space of $L^{\omega}(\Omega, X)$ is $\bigcup_{1<q \leq \infty} L^{q}\left(\Omega, X^{*}\right)$; if $X$ is reflexive, then $L^{\omega}(\Omega, X)$ also is reflexive.

\section{Preliminaries}

Let $(\Omega, \Sigma, \mu)$ be a measure space with finite measure; let $L^{0}(\Omega)$ be the classes of all measurable functions on $\Omega$.

Throughout this paper, $X$ stands for a fixed initial Banach algebra with the norm $\|\cdot\|_{X}$. By $L^{0}(\Omega, X)$ we denote the classes of all measurable by Bochner mappings from $\Omega$ to $X$; that is,

$$
L^{0}(\Omega, X)=\left\{f: \Omega \longrightarrow X:\|f(\omega)\|_{X} \in L^{0}(\Omega)\right\} .
$$

It is known [11] that $L^{0}(\Omega, X)$ is algebra with respect to multiplication operation $(f g)(\omega)=f(\omega) g(\omega)$ for any 
$f, g \in L^{0}(\Omega, X)$. By $L^{p}(\Omega, X), 1 \leq p<\infty$, we denote the Banach space of $X$ valued measurable functions $f$ on $(\Omega, \Sigma, \mu)$ with the norm defined as

$$
\|f\|_{p}=\left(\int_{\Omega}\|f(\omega)\|_{X}^{p} d \mu\right)^{1 / p}=\left|\|f(\omega)\|_{X}\right|_{p} ;
$$

that is, $L^{p}(\Omega, X)=\left\{f \in L^{0}(\Omega, X):\|f(\omega)\|_{X} \in L^{p}\right\}$ and $L^{\infty}(\Omega, X)=\left\{f \in L^{0}(\Omega, X): \exists \lambda>0,\|f(\omega)\|_{X} \leq \lambda\right.$ a.e. $\}=$ $\left\{f \in L^{0}(\Omega, X):\|f(\omega)\|_{X} \in L^{\infty}\right\}$ with the norm defined as

$$
\|f\|_{\infty}=\inf \left\{\lambda>0:\|f(\omega)\|_{X} \leq \lambda \text { a.e. }\right\} .
$$

It is known [11] that the pair $\left(L^{\infty}(\Omega, X),\|\cdot\|_{\infty}\right)$ is a Banach algebra.

The following well known properties hold true.

(a) For $f, g \in L^{p}(\Omega, X)$ the Minkowski inequality

$$
\|f+g\|_{p} \leq\|f\|_{p}+\|g\|_{p}
$$

is valid.

(b) For $h \in L^{p}$ and $f \in L^{q}(\Omega, X)$, where $q:=p /(p-1)$ for $p \neq 1$ and $q:=\infty$ for $p=1$, the Hölder inequality

$$
\|h f\|_{1} \leq|h|_{p}\|f\|_{q}
$$

is valid.

Proposition 1. (i) If $f \in L^{\infty}(\Omega, X)$ and $g \in L^{p}(\Omega, X)$ then $f g \in L^{p}(\Omega, X)$ and

$$
\|f g\|_{p} \leq\|f\|_{\infty}\|g\|_{p} .
$$

(ii) If $p, q, r \geq 1,1 / r=(1 / p)+(1 / q), f \in L^{p}(\Omega, X)$, and $g \in L^{q}(\Omega, X)$, then $f g \in L^{r}(\Omega, X)$ and $\|f g\|_{r} \leq\|f\|_{p}\|g\|_{q}$.

(iii) If $\|f(\omega)\|_{X} \leq\|g(\omega)\|_{X}$ a.e., $f \in L^{0}(\Omega, X)$, and $g \in$ $L^{p}(\Omega, X)$, then $f \in L^{p}(\Omega, X)$ and $\|f\|_{p} \leq\|g\|_{p}$.

Proof. (i) Let $f \in L^{\infty}(\Omega, X)$ and $g \in L^{p}(\Omega, X)$. Since $\|f(\omega) g(\omega)\|_{X} \leq\|f(\omega)\|_{X}\|g(\omega)\|_{X} \leq\|f\|_{\infty}\|g(\omega)\|_{X}$ and $\|g(\omega)\|_{X} \in L^{p}$ we have that $f g \in L^{p}(\Omega, X)$ and

$$
\begin{aligned}
\|f g\|_{p} & =\left(\int_{\Omega}\|f(\omega) g(\omega)\|_{X}^{p} d \mu\right)^{1 / p} \\
& \leq\|f\|_{\infty}\left(\int_{\Omega}\|g(\omega)\|_{X}^{p} d \mu\right)^{1 / p}=\|f\|_{\infty}\|g\|_{p} .
\end{aligned}
$$

(ii) Let $p, q, r \geq 1,1 / r=(1 / p)+(1 / q), f \in L^{p}(\Omega$, $X)$, and $g \in L^{q}(\Omega, X)$. As $\|f(\omega)\|_{X} \in L^{p}$ and $\|g(\omega)\|_{X} \in$ $L^{q}$ from inequality $\|f(\omega) g(\omega)\|_{X} \leq\|f(\omega)\|_{X}\|g(\omega)\|_{X}$ we get that $f g \in L^{r}(\Omega, X)$ and $\|f g\|_{r}=$ $\left(\int_{\Omega}\|f(\omega) g(\omega)\|_{X}^{r} d \mu\right)^{1 / r} \leq\left(\int_{\Omega}\left(\|f(\omega)\|_{X}\|g(\omega)\|_{X}\right)^{r} d \mu\right)^{1 / r} \leq$ $\left(\int_{\Omega}\|f(\omega)\|_{X}^{p} d \mu\right)^{1 / p}\left(\int_{\Omega}\|g(\omega)\|_{X}^{q} d \mu\right)^{1 / q}=\|f\|_{p}\|g\|_{q}$.

(iii) Let $\|f(\omega)\|_{X} \leq\|g(\omega)\|_{X}$ a.e., $f \in L^{0}(\Omega, X)$, and $g \in$ $L^{p}(\Omega, X)$. Then $\int_{\Omega}\|f(\omega)\|_{X}^{p} d \mu \leq \int_{\Omega}\|g(\omega)\|_{X}^{p} d \mu<\infty$. This means that $f \in L^{p}(\Omega, X)$ and $\|f\|_{p} \leq\|g\|_{p}$.

\section{The Arens Algebras $L^{\omega}(\Omega, X)$ of Vector-Valued Functions}

In this section we introduce notion of the Arens algebras of vector-valued functions. Put

$$
L^{\omega}(\Omega, X)=\bigcap_{p \geq 1} L^{p}(\Omega, X) ;
$$

that is, $L^{\omega}(\Omega, X)=\left\{f \in L^{0}(\Omega, X):\|f\|_{1}<\infty,\|f\|_{2}<\right.$ $\left.\infty, \ldots,\|f\|_{p}<\infty, \ldots\right\}$, and will consider in $L^{\omega}(\Omega, X)$ local convex topology $\tau_{X}$ is generated by system of norms $\left\{\|\cdot\|_{p}\right\}_{p \geq 1}$. From (ii) Proposition 1 we get that $\|f\|_{1} \leq\|f\|_{2} \leq$ $\cdots \leq\|f\|_{n} \leq \cdots$, that is, the topology $\tau_{X}$ generated by countable system of norms $\left\{\|\cdot\|_{n}\right\}_{n=1}^{\infty}$. By Theorem III.2.2 [12] it means that topological vector space $\left(L^{\omega}(\Omega, X), \tau_{X}\right)$ is metrizable space with respect to the metric

$$
\rho(f, g)=\sum_{k=1}^{\infty} \frac{1}{2^{k}} \frac{\|f-g\|_{k}}{1+\|f-g\|_{k}} .
$$

Theorem 2. $\left(L^{\omega}(\Omega, X), \tau_{X}\right)$ is a complete topological algebra.

Proof. Let $\left\{f_{n}\right\}$ be a fundamental sequence in $\left(L^{\omega}(\Omega, X), \tau_{X}\right)$. Then $\left\{f_{n}\right\}$ is fundamental in $L^{p}(\Omega, X)$ for any $1 \leq p<\infty$. Since $L^{p}(\Omega, X)$ is complete there exist $f_{p} \in L^{p}(\Omega, X)$ such that $\left\|f_{n}-f_{p}\right\|_{p} \rightarrow 0$ as $n \rightarrow \infty$, as

$$
\left\|f_{n}-f_{p}\right\|_{q} \leq \mu(\Omega)^{p q / p+q}\left\|f_{n}-f_{p}\right\|_{p},
$$

for all $1 \leq q<p<\infty$, implies that $\left\|f_{n}-f_{p}\right\|_{q} \rightarrow 0$. This means that $f_{p}=f_{q}$ if $1 \leq q<p<\infty$. Therefore $f=f_{p}$ is limit of sequence $\left\{f_{n}\right\}$ and $f$ is an element of space $L^{\omega}(\Omega, X)$. Since the inequality

$$
\|f g\|_{p} \leq\|f\|_{2 p}\|g\|_{2 p}
$$

is valid the multiplication is continuous on $L^{\omega}(\Omega, X)$. Hence $\left(L^{\omega}(\Omega, X), \tau_{X}\right)$ is a topological algebra.

Theorem 3. (i) If $f \in L^{0}(\Omega, X), g \in L^{\omega}(\Omega, X)$, and $\|f(\omega)\|_{X} \leq\|g(\omega)\|_{X}$ a.e. then $f \in L^{\omega}(\Omega, X)$ and $\rho(f, 0) \leq$ $\rho(g, 0)$.

(ii) If $g \in L^{\infty}(\Omega, X)$ and $f \in L^{\omega}(\Omega, X)$ then $g f \in$ $L^{\omega}(\Omega, X)$ and there exist $m \in \mathbb{N}$

$$
\rho(g f, 0) \leq 2^{m} \rho(f, 0) .
$$

Proof. (i) As $g \in L^{\omega}(\Omega, X)$ we have that $g \in L^{p}(\Omega, X)$ for any $p \geq 1$. Then from $\|f(\omega)\|_{X} \leq\|g(\omega)\|_{X}$ a.e. by Proposition 1(iii) we get that $f \in L^{p}(\Omega, X)$ for any $p \geq 1$. This means that $f \in L^{\omega}(\Omega, X)$. Since $\|f\|_{p} \leq\|g\|_{p}$ for any $p \geq 1$ we have

$$
\rho(f, 0)=\sum_{k=1}^{\infty} \frac{1}{2^{k}} \frac{\|f\|_{k}}{1+\|f\|_{k}} \leq \sum_{k=1}^{\infty} \frac{1}{2^{k}} \frac{\|g\|_{k}}{1+\|g\|_{k}}=\rho(g, 0) .
$$

(ii) Let $g \in L^{\infty}(\Omega, X)$ and $f \in L^{\omega}(\Omega, X)$; then $f \in$ $L^{p}(\Omega, X)$ for all $p \geq 1$. By Proposition 1(i) $g f \in L^{p}(\Omega, X)$ 
for all $p \geq 1$. Therefore $g f \in L^{\omega}(\Omega, X)$. We chose $m \in \mathbb{N}$ that $\|g\|_{\infty} \leq 2^{m}$. Then using Proposition 1(i) we get

$$
\begin{aligned}
\rho(g f, 0) & =\sum_{k=1}^{\infty} \frac{1}{2^{k}} \frac{\|g f\|_{k}}{1+\|g f\|_{k}} \leq \sum_{k=1}^{\infty} \frac{1}{2^{k}} \frac{\|g\|_{\infty}\|f\|_{k}}{1+\|g\|_{\infty}\|f\|_{k}} \\
& \leq \sum_{k=1}^{\infty} \frac{1}{2^{k}} \frac{2^{m}\|f\|_{k}}{1+2^{m}\|f\|_{k}} \leq 2^{m} \sum_{k=1}^{\infty} \frac{1}{2^{k}} \frac{\|f\|_{k}}{1+\|f\|_{k}} \\
& =2^{m} \rho(f, 0) .
\end{aligned}
$$

Theorem 4. $L^{\infty}(\Omega, X)$ is dense in $L^{\omega}(\Omega, X)$ and $L^{\omega}(\Omega, X)$ is dense in $L^{p}(\Omega, X)$.

Proof. Let $f \in L^{\omega}(\Omega, X)$. There exists sequence of idempotents $e_{n}$ from $L^{0}(\Omega)$ such that $e_{n} \uparrow \mathbf{1}$, where $\mathbf{1}$ is unit element on $L^{0}(\Omega)$. Then $\left\|f(\omega) e_{n}(\omega)\right\|_{X}=\|f(\omega)\|_{X} e_{n}(\omega) \in L^{\infty}(\Omega)$; that is, $f(\omega) e_{n}(\omega) \in L^{\infty}(\Omega, X)$. Let $f_{n}=f e_{n}$. Then $f_{n} \in$ $L^{\infty}(\Omega, X)$ and $\left\|f(\omega)-f_{n}(\omega)\right\|_{X}=\left\|f(\omega)-f(\omega) e_{n}(\omega)\right\|_{X}=$ $\left\|f(\omega)\left(\mathbf{1}(\omega)-e_{n}(\omega)\right)\right\|_{X}=\|f(\omega)\|_{X}\left(\mathbf{1}(\omega)-e_{n}(\omega)\right) \downarrow 0$. As norm $|\cdot|_{p}$ is order continuous in $L^{p}$ space for all $p \geq 1$ we get that $\left\|f-f_{n}\right\|_{p}=\left|\|f(\omega)\|_{X}\left(\mathbf{1}(\omega)-e_{n}(\omega)\right)\right|_{p} \rightarrow 0$ for all $p \geq 1$ as $n \rightarrow \infty$. Therefore

$$
\rho\left(f_{n}, f\right)=\sum_{k=1}^{\infty} \frac{1}{2^{k}} \frac{\left\|f_{n}-f\right\|_{k}}{1+\left\|f_{n}-f\right\|_{k}} \longrightarrow 0
$$

as $n \rightarrow \infty$. The density $L^{\omega}(\Omega, X)$ in $L^{p}(\Omega, X)$ follows from density $L^{\infty}(\Omega, X)$ in $L^{\omega}(\Omega, X)$.

Let $\varphi_{1}, \varphi_{2}, \ldots, \varphi_{n} \in L^{\omega}$ and $x_{1}, x_{2}, \ldots, x_{n} \in X$. Then we can define function $f: \Omega \rightarrow X$ by setting

$$
f(\omega)=\sum_{i=1}^{n} \varphi_{i}(\omega) x_{i}
$$

for almost all $\omega \in \Omega$.

We will denote $L^{\omega} \otimes X=\left\{f \in L^{0}(\Omega, X): f(\omega)=\right.$ $\left.\sum_{i=1}^{n} \varphi_{i}(\omega) x_{i}, \varphi_{i} \in L^{\omega}, x_{i} \in X, i=\overline{1, n}, n \in \mathbb{N}\right\}$. $L^{\omega} \otimes X$ is subspace of $L^{\omega}(\Omega, X)$, because $\int_{\Omega}\|f(\omega)\|_{X}^{p} d \mu \leq$ $\sum_{i=1}^{n} \int_{\Omega}\left|\varphi_{i}(\omega)\right|^{p}\left\|x_{i}\right\|_{X}^{p} d \mu=\sum_{i=1}^{n}\left\|x_{i}\right\|_{X}^{p} \int_{\Omega}\left|\varphi_{i}(\omega)\right|^{p} d \mu<\infty$ for all $p \geq 1$.

Theorem 5. $L^{\omega} \otimes X$ is dense in $L^{\omega}(\Omega, X)$.

Proof. Let $f \in L^{\omega}(\Omega, X)$. Then $f \in L^{p}(\Omega, X)$ for all $p \geq 1$.

Let $s_{n}(\omega)$ be the sequence simple $X$ valued functions; that is, every $s_{n}(\omega)$ has form $\left\{\sum_{i=1}^{m} \chi_{B_{i}}(\omega) x_{i}: B_{i} \in \sum, B_{i} \cap B_{j}=\right.$ $\left.\emptyset, x_{i} \in X\right\}$, such that $s_{n}(\omega) \rightarrow f(\omega)$ a.e. on $\Omega$. Then $\left\|s_{n}(\omega)\right\|_{X} \rightarrow\|f(\omega)\|_{X}$ almost everywhere on $\Omega$.

Let $A_{n}=\left\{\omega:\left\|s_{n}(\omega)\right\|_{X} \leq 2\|f(\omega)\|_{X}\right\}$. If we set $g_{n}(\omega)=$ $s_{n}(\omega) \chi_{A_{n}}(\omega)$ we have that $g_{n}(\omega) \rightarrow f(\omega)$ a.e. on $\Omega$ and

$$
\begin{aligned}
\sup _{n}\left\|g_{n}(\omega)-f(\omega)\right\|_{X} & \leq \sup _{n}\left\|g_{n}(\omega)\right\|_{X}+\|f(\omega)\|_{X} \\
& \leq 3\|f(\omega)\|_{X} .
\end{aligned}
$$

By dominated convergence we have that

$$
\int_{\Omega}\left\|g_{n}(\omega)-f(\omega)\right\|_{X}^{p} d \mu \longrightarrow 0,
$$

for all $p \geq 1$ and of course $g_{n} \in L^{\omega} \otimes X$.

\section{Dual of Arens Algebras $L^{\omega}(\Omega, X)$}

In this section we study dual of Arens algebras of vectorvalued functions. It is shown that if dual of $X$ has Radon-Nikodym property, then dual space of $L^{\omega}(\Omega, X)$ is $\bigcup_{1<q \leq \infty} L^{q}\left(\Omega, X^{*}\right)$; if $X$ is reflexive, then $L^{\omega}(\Omega, X)$ also is reflexive.

Definition 6 (see [13]). Recall that a Banach space $Y$ is said to possess the Radon-Nikodym property, with respect to $\mu$, if for any $\mu$-continuous vector measure $m: \Sigma \rightarrow Y$ of bounded total variation, there exists a Bochner integrable function $f$ : $\Omega \rightarrow Y$ such that

$$
m(A)=\int_{A} f d \mu,
$$

for all $A \in \Sigma$. It is known [13] that dual of Banach algebra $c_{0}$, $c_{0}^{*}=l_{1}$ has the Radon-Nikodym property.

A class of Banach algebras $X$ whose duals $X^{*}$ have the Radon-Nikodym property is studied in [14].

Theorem 7. If the dual space $X^{*}$ of Banach algebra $X$ has Radon-Nikodym property with respect to $\mu$, then the dual of $L^{\omega}(\Omega, X)$ is the space

$$
E=\bigcup_{1<q \leq \infty} L^{q}\left(\Omega, X^{*}\right)
$$

and for any $\ell \in\left(L^{\omega}(\Omega, X)\right)^{*}$ there exists $g \in E$ such that

$$
\ell(f)=\int_{\Omega} g f d \mu\left(f \in L^{\omega}(\Omega, X)\right) .
$$

Proof. Since $L^{\omega}(\Omega, X)$ is countably normed space by [15] dual of $L^{\omega}(\Omega, X)$ is the space $\bigcup_{1<q \leq \infty}\left(L^{p}(\Omega, X)\right)^{*}$, where $(1 / p)+$ $(1 / q)=1$. As $X^{*}$ has the Radon-Nikodym property with respect to $\mu$ by Theorem IV.1.1 $[13]\left(L^{p}(\Omega, X)\right)^{*}=L^{q}\left(\Omega, X^{*}\right)$, where $(1 / p)+(1 / q)=1$. Hence, the dual of $L^{\omega}(\Omega, X)$ is the space

$$
E=\bigcup_{1<q \leq \infty} L^{q}\left(\Omega, X^{*}\right)
$$

where $(1 / p)+(1 / q)=1$.

Let $\ell$ be a continuous linear functional on $L^{\omega}(\Omega, X)$. Then by [15] there exists natural number $p(1 \leq p<\infty)$ and $C>0$ such that

$$
|\ell(f)| \leq C\|f\|_{p} .
$$

It means $\ell \in\left(L^{p}(\Omega, X)\right)^{*}$. Using the general form of continuous linear functionals in $L^{p}(\Omega, X)$ (see [13], IV.1) we get that there exists $g \in L^{q}\left(\Omega, X^{*}\right) \subset E$ such that

$$
\ell(f)=\int_{\Omega} g f d \mu .
$$


Theorem 8. If $X$ is a reflexive Banach algebra then $L^{\omega}(\Omega, X)$ is reflexive.

Proof. Let $X$ be a reflexive Banach algebra. Since every reflexive space has Radon-Nikodym property with respect to $\mu$, by Theorem 7 ,

$$
\begin{aligned}
L^{\omega}(\Omega, X)^{* *} & =\left(\bigcup_{1<q \leq \infty} L^{q}\left(\Omega, X^{*}\right)\right)^{*}=\bigcap_{p \geq 1} L^{p}\left(\Omega, X^{* *}\right) \\
& =\bigcap_{p \geq 1} L^{p}(\Omega, X)=L^{\omega}(\Omega, X) .
\end{aligned}
$$

This means that $L^{\omega}(\Omega, X)$ is semireflexive space. By Theorem $2 L^{\omega}(\Omega, X)$ is $(F)$-space. Since by Corollary II.7.1 [16] every $(F)$-space is barreled we have that $L^{\omega}(\Omega, X)$ is barreled. Hence by IV.5. [16] $L^{\omega}(\Omega, X)$ is reflexive.

\section{Conflict of Interests}

The authors declare that there is no conflict of interests regarding the publication of this paper.

\section{Acknowledgment}

The first author (I. G. Ganiev) acknowledges the MOHE Grant FRGS13-071-0312.

\section{References}

[1] R. Arens, "The space $L^{\omega}$ and convex topological rings," Bulletin of the American Mathematical Society, vol. 52, pp. 931-935, 1946.

[2] G. R. Allan, "On a class of locally convex algebras," Proceedings of the London Mathematical Society, vol. 17, no. 3, pp. 91-114, 1967.

[3] S. J. Bhatt, "On Arens' algebra $L^{\omega}$," Glasnik Matematički, vol. 15(35), no. 2, pp. 305-314, 1980.

[4] S. J. Bhatt, “On Arens' algebra $L^{\omega}$. II," Glasnik Matematički, vol. 16(36), no. 2, pp. 297-306, 1981.

[5] A. Inoue, "On a class of unbounded operator algebras. II," Pacific Journal of Mathematics, vol. 66, no. 2, pp. 411-431, 1976.

[6] B. S. Zakirov, "Noncommutative Arens algebras," Uzbek Mathematical Journal, no. 1, pp. 17-24, 1997 (Russian).

[7] R. Z. Abdullaev, "Spaces dual to noncommutative Arens algebras,” Uzbek Mathematical Journal, no. 2, pp. 3-7, 1997 (Russian).

[8] R. Z. Abdullaev and V. I. Chilin, "Arens algebras, associated with commutative von Neumann algebras," Annales Mathématiques Blaise Pascal, vol. 5, no. 1, pp. 1-12, 1998.

[9] A. A. Alimov, "Arens algebras associated with a center-valued trace," Uzbek Mathematical Journal, no. 1, pp. 12-18, 2000 (Russian).

[10] A. A. Alimov, "Arens algebras ofmeasurable operators for Maharam traces," http://arxiv.org/abs/1307.2751v1.

[11] V. I. Chilin, I. G. Ganiev, and K. K. Kudaĭbergenov, "The Gelfand-Naimark theorem for $C^{*}$-algebras-algebras over a ring of measurable functions," Russian Mathematics, vol. 52, no. 2, pp. 58-66, 2008.
[12] L. V. Kantorovich and G. P. Akilov, Functional Analysis, vol. 14, Pergamon Press, Oxford, UK, 2nd edition, 1982.

[13] J. Diestel and J. J. Uhl Jr., Vector Measures, American Mathematical Society, Providence, RI, USA, 1977.

[14] H. S. Mustafayev, "A class of Banach algebras whose duals have the Radon-Nikodym property," Archiv der Mathematik, vol. 87, no. 5, pp. 449-457, 2006.

[15] B. Z. Vulikh, Introduction to Functional Analysis, Pergamon Press, New York, NY, USA, 1963.

[16] H. H. Schaefer, Topological Vector Spaces, Springer, New York, NY, USA, 1971. 


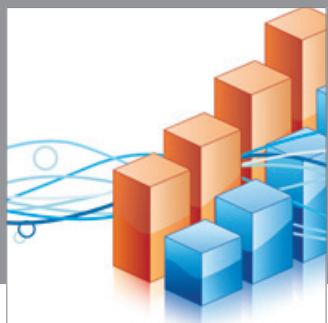

Advances in

Operations Research

mansans

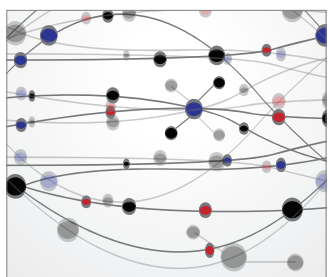

The Scientific World Journal
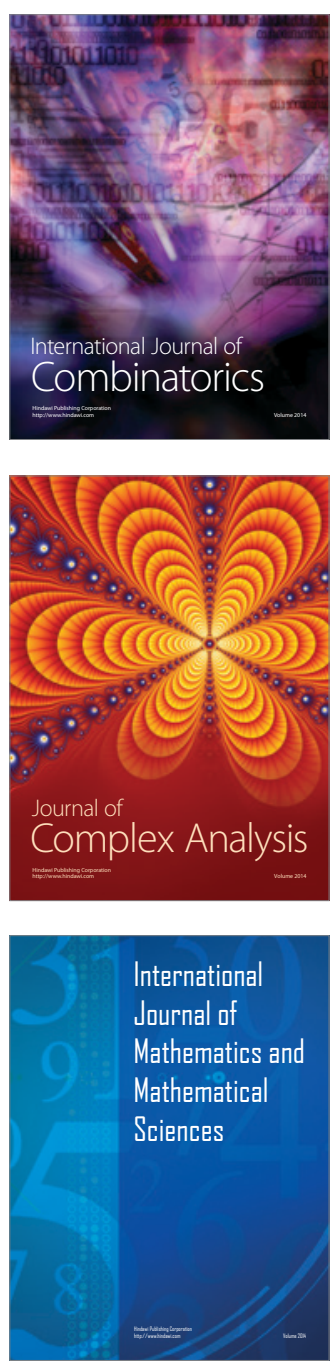
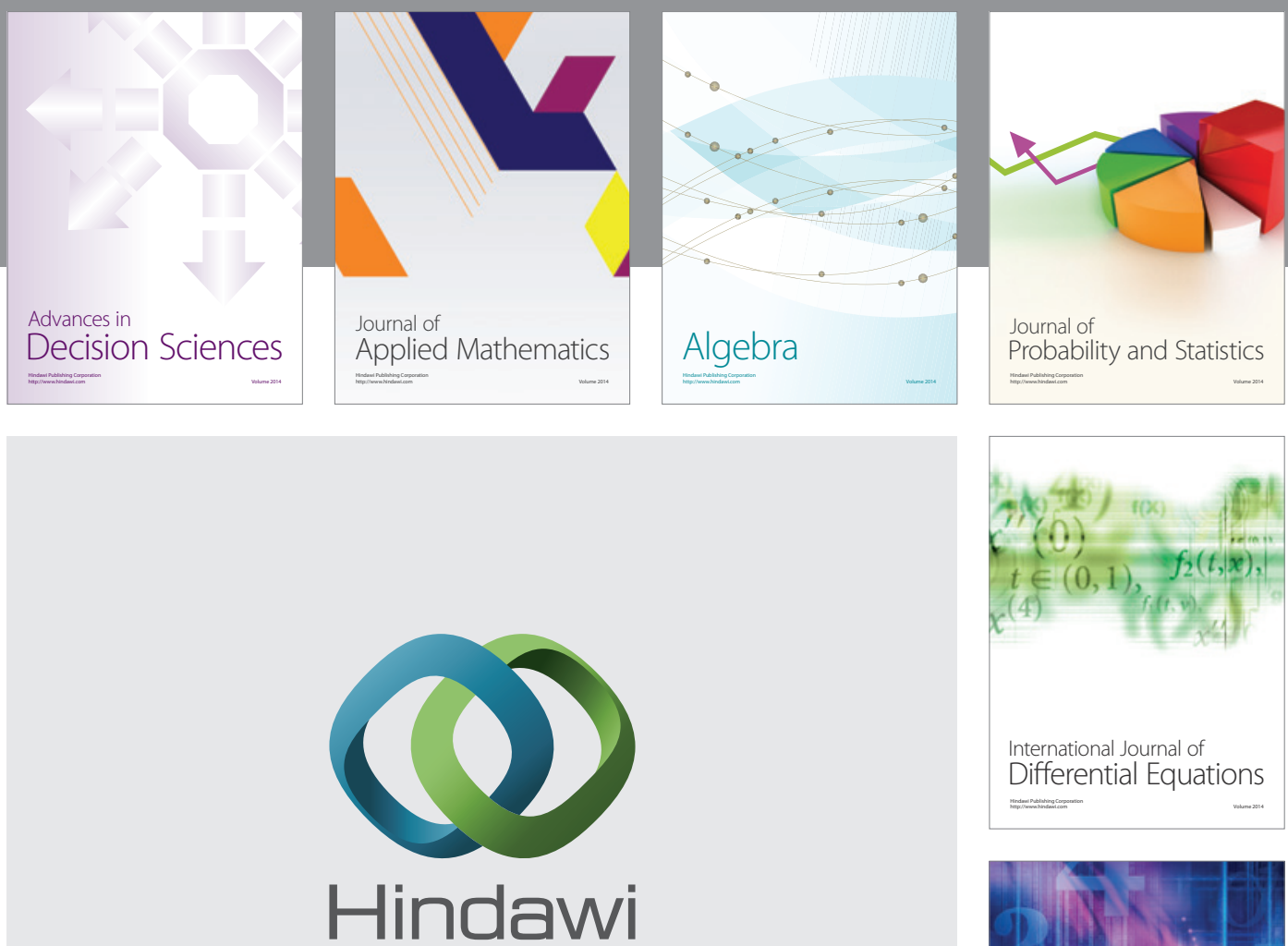

Submit your manuscripts at http://www.hindawi.com
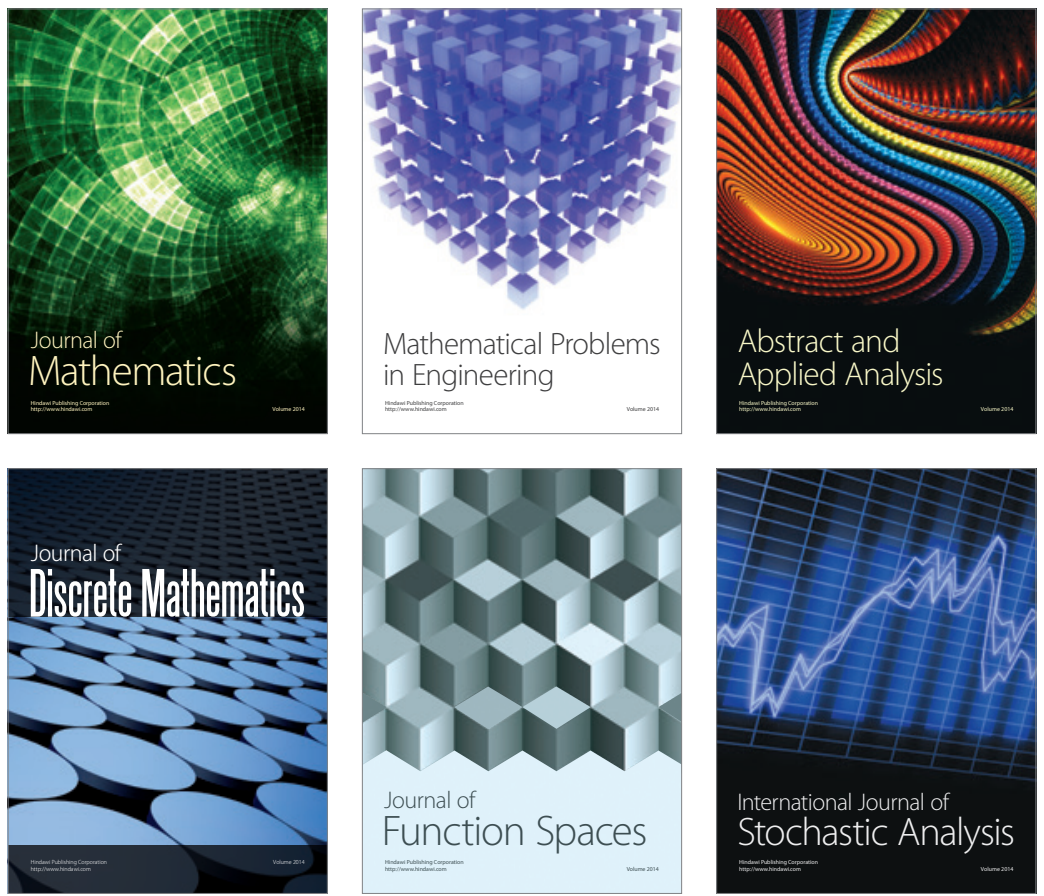

Journal of

Function Spaces

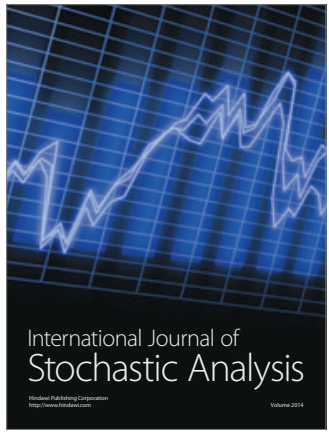

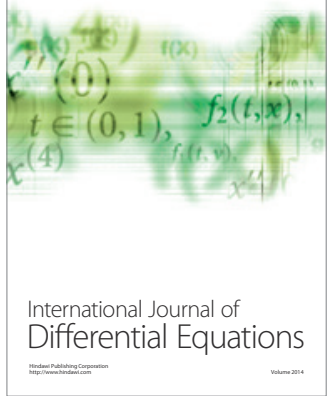
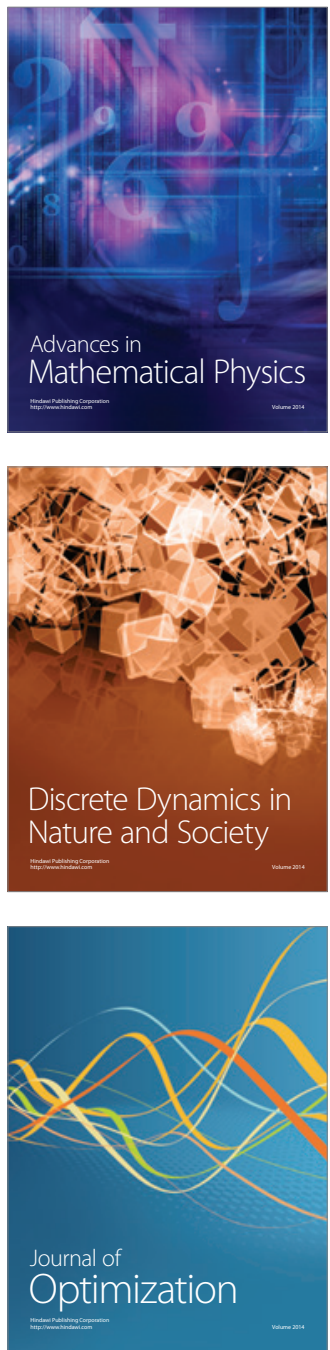